\title{
Effects of subject-generated stories on recall
}

\author{
GLENN GAMST and JOEL S. FREUND \\ University of Arkansas, Fayetteville, Arkansas 72701
}

\begin{abstract}
Two free recall experiments that used modified narrative chaining instructions are reported. The first experiment compared instructions to create a story with standard free recall instructions. Subjects were given 5 min to study a 30-item list of words for later recall. One group was told to create a story with the words; the other group was simply told to study them. Mean recall did not differ as a function of instructions. In the second experiment, study time was manipulated in an attempt to eliminate the possibility that subjects in all conditions were creating stories. Again recall of the subjects given story instructions did not differ from those given standard free recall instructions. The results of the experiments are discussed in terms of methodological differences between these experiments and those of other investigators.
\end{abstract}

Craik and Lockhart (1972) and Craik and Tulving (1975) proposed a framework for the investigation of human memory in which information is presumed to be encoded at varying levels of analysis. These levels refer to the amount of semantic or cognitive elaboration with which a memory trace is imbued, with the traces receiving more elaborate encoding being better recalled.

Many of the studies supporting a levels-of-processing analysis have utilized incidental learning paradigms involving various types of orienting tasks. In particular, Craik and his associates have typically compared tasks that focus on the extremes of the "depth" dimension. That is, the tasks focus the subject's attention either on nonsemantic (orthographic, rhyming, or typeface) attributes or on semantic attributes. However, Craik and Lockhart (1972) clearly state that increasing the amount of semantic elaboration within a semantic task should also lead to better recall.

A technique which apparently increases the amount of semantic elaboration within a semantic task is the narrative chaining task introduced by Bower and Clark (1969). This technique not only appears to increase semantic elaboration, but it also produces better recall than methods involving less elaboration. For example, Bower and Clark presented subjects 12 serial lists of 10 words each. Immediate recall of each list produced a median recall of $99.9 \%$ for the narrative chaining group and $99.1 \%$ for the yoked control. An end-of-session cued-recall test of all lists produced median recall of $93 \%$ and $13 \%$ for the narrative chaining and control subjects, respectively.

Several other investigators (Herrmann, Geisler, \& Atkinson, 1973; Light \& Schurr, 1973; Santa, Ruskin, \& Yio, 1973) have used narrative chaining instructions and,

The present research was in partial fulfillment of the requirements for the Master of Arts degree for the first author. Requests for reprints should be sent to Joel S. Freund, Department of Psychology, University of Arkansas, Fayetteville, Arkansas 72701. in general, found better recall and recognition than with standard instructions. These results are consistent with a levels-of-processing notion, if one assumes that incorporating a word into a story requires more elaborate processing of that word than does simply retaining it.

The narrative chaining method typically requires subjects to learn several rather short lists. Bower and Clark (1969) used 12 10-item lists; Hermann et al. (1973) used 8 8-item and 8 16-item lists; Light and Schurr (1973) used 96 words divided into 128 -item blocks or, in essence, 12 8-item lists; Santa et al. (1973) used 6 10-item lists. The present experiments were designed primarily to extend the results of the narrative chaining technique to relatively long lists of words. The intention was to interpret the results within the levelsof-processing framework.

However, since the present experiments were undertaken, a slightly different interpretation of the processes underlying narrative chaining instructions has been proposed by Bellezza and his associates (Bellezza, Chessman, \& Reddy, 1977; Bellezza, Richards, \& Geiselman, 1976). Bellezza contends that the narrative chaining technique leads to better recall because it leads to better organization among the presented words, not to greater semantic elaboration. He further stated, "Once a word has been semantically processed to the point of comprehension, further semantic processing or elaboration has a relatively small effect on improving recall performance compared to the effect of organizing a word through the use of some mnemonic device" (1977, p. 540).

While the present experiments were designed to be interpreted according to a levels-of-processing framework, they can also be applied to an organizational analysis. In Experiment 1, a group given narrative chaining instructions was compared to a group given standard free recall instructions. According to either interpretation, organization or semantic elaboration, the narrative chaining technique should produce better recall. 


\section{EXPERIMENT 1}

\begin{abstract}
Method
Design and subjects. The design was a 2 by 3 factorial with two word lists (concrete, abstract) and three types of encoding instructions (standard free recall, story recall, story). The subjects were 90 undergraduates serving in the experiment as partial fulfillment of a course requirement. Subjects were tested in small groups until there were 15 subjects in each condition. Subjects in each group read one of the three types of instructions; the particular instructions given to any group were determined from a block randomized schedule. Approximately one-half of the subjects in each group were given each list (concrete, abstract).
\end{abstract}

Word lists. Two lists were created from nouns taken from the Paivio, Yuille, and Madigan (1968) norms. The concrete list consisted of 30 high-imagery nouns (mean $I=6.09$ ) with mean concreteness, meaningfulness, and frequency ratings of 6.32 , 5.57 , and 41.90 , respectively. The abstract list contained 30 low-imagery nouns (mean $I=2.65$ ) with concreteness, meaningfulness, and frequency ratings of $2.55,5.47$, and 33.07. Each list was typed in a random order on an $8 \frac{1}{2} \times 11$ in. sheet of paper for presentation to the subjects.

Procedure. At the beginning of a session, subjects read appropriate instructions and the study sheets were distributed. All subjects were given 5 min to study the list, after which they were asked to recall as many words as possible. The three encoding conditions differed in terms of the encoding instructions and the nature of the recall test. Subjects in the free recall condition were given standard free recall instructions and were asked to recall the words in any order. Subjects in the story recall and story conditions were instructed to study the words by incorporating them into a story. The two conditions differed only in the manner in which subjects recalled the words. Subjects in the story recall condition were asked to write the stories they had devised, circling the words they thought were on the study list. In the story condition, subjects were instructed to write all the words they could remember from the word list. After recalling as many as they could, subjects were instructed to write the stories they had devised, circling the target words.

\section{Results and Discussion}

The mean number of words recalled and the standard deviations for all conditions of Experiment 1 are presented in Table 1. Recall was not enhanced by the presumably more elaborate encoding instructions (i.e., story instructions). The three encoding tasks did not differ reliably $[\mathrm{F}(2,84)<1]$. While the main effect of imagery was reliable $[F(1,84)=85.59]$, the Encoding Task by Imagery interaction was not $[\mathrm{F}(2,84)=2.75]$. The MSe for all Fs was 19.85.

Although many possible explanations for the failure of the results to conform to predictions can be offered,

Table 1

Mean Total Recall as a Function of Instructions and Imagery

\begin{tabular}{lccccc}
\hline & \multicolumn{4}{c}{ Imagery } \\
\cline { 2 - 3 } \cline { 5 - 6 } Condition & Mean & SD & & Mean & SD \\
\cline { 2 - 3 } \cline { 5 - 6 } Low & & & \multicolumn{2}{c}{ High } \\
\hline Free Recall & 13.13 & 4.17 & & 20.33 & 5.45 \\
Story & 10.13 & 3.66 & & 21.93 & 4.06 \\
Story Recall & 13.73 & 4.46 & & 20.80 & 4.71 \\
\hline
\end{tabular}

only two will be examined here. First, it is possible that the instructions to construct a story did not result in more semantic elaboration or more organization than did standard instructions. Second, it is possible that the instructions did enhance semantic elaboration or organization but that sufficient study time allowed the free recall subjects to achieve the same level of processing or organization. More specifically, perhaps the free recall subjects made up their own stories.

\section{EXPERIMENT 2}

The second experiment was an examination of the possibility that, given enough study time, all subjects would process to the same level. However, if study time was limited, subjects given free recall instructions would use less elaborate rehearsal methods or less effective organization, while subjects given instructions to generate a story should use more elaborate methods of processing. Thus, with limited study time, instructions to create a story should be more effective than free recall instructions, while, with longer study intervals, the two should not differ. Further, if the time allowed for study was brief enough, a group given a story to study might benefit the most, because the story could lead to deeper levels of processing or better organization more quickly than instructions to construct a story.

\section{Method}

Design and subjects. The design was a 3 by 3 factorial with three methods of encoding (free recall, story, intact) and three study intervals $(1,3,5 \mathrm{~min})$. The subjects were 180 undergraduates serving in the experiment in partial fulfillment of a course requirement. Subjects were run in groups of five, with assignment of groups to conditions accomplished by a process of block randomization.

Materials. The concrete list used in Experiment 1 was again used. The 10 stories used in the intact condition were generated by the subjects in Experiment 1 . The punctuation and grammar were corrected, and any omitted target words were replaced without changing the content of the story. The stories were then typed, with each target word underlined and printed in uppercase letters. Ten different orders of the words were constructed for use in the free recall and story conditions. Each order corresponded to the order of the target words in one of the stories used in the intact condition.

Procedure. Subjects were either told to study the words and recall them in any order they wished (free recall) or asked to think up a story using the target words and at recall told to write down all the words they could remember (story). In the intact condition, each subject was given one of the 10 stories with the explanation that the story had been written by a subject in a previous experiment. The subjects were then instructed to study the capitalized and underlined words, using the story to help them remember the words. After the appropriate study periods, subjects were asked to recall as many words as they could remember.

\section{Results and Discussion}

Percent recall increased as a function of study time for all encoding tasks $[\mathrm{F}(2,171)=67.33, \mathrm{p}<.01, \mathrm{MSe}=$ 19.96]. In the free recall condition the mean percent 
recall was $33.3,53.3$, and 70.0 , for the 1-, 3-, and 5-min intervals, respectively. The same intervals produced percent recalls of $37.2,59.3,62.5$ and $32.8,50.2,64.3$ in the story and intact conditions, respectively. Neither the main effect of the encoding task nor the interaction of Study Interval by Encoding Task was significant $[F(2,171)=1.15$ and $F(4,171)=1.35$, ps $>.05$, respectively]. From these results, it appears that limiting the study time did not have the hypothesized effect. Subjects given standard free recall instructions recalled as many words as subjects given instructions to construct a story. One implication of these results is that the free recall subjects were not constructing stories; whatever process or strategy they employed, it was as effective as constructing a story.

\section{GENERAL DISCUSSION}

Although the present experiments did not replicate the results of previous investigators (Bellezza et al., 1976, 1977; Hermann et al., 1973; Light \& Schurr, 1973; Santa et al, 1973), the present results should not be dismissed as a statistical aberration. At least one plausible explanation could account for all the results.

A comparison of the present study with all previous studies, except those of Bellezza et al. $(1976,1977)$, reveals two major differences: list length and number of lists. While we used a single long list, previous investigators used several relatively short lists, a fact that is relevant if we make some simple assumptions.

We must assume that in order to construct a story from a list of unrelated words, a subject must somehow retain the story line in immediate memory, a task most readily accomplished by covertly rehearsing the story. It is also reasonable to assume that this task is rather difficult and subjects would require some practice before becoming proficient at it. (This assumption could be tested by examining the recall of the narrative chaining and free recall groups across stage of practice. The narrative chaining groups should show a greater improvement over lists than the free recall groups.) Subjects in the present experiments learned only one list, and thus the advantage of the story mnemonic was not demonstrated.

In contrast to the previous studies, Bellezza et al. $(1976,1977)$ used a single long list of words and found better recall for the story-mnemonic conditions. However, their procedures differed from ours in several important ways, which may account for their finding the effect when we did not. In two of their six experiments (Experiment 1, 1976; Experiment 2, 1977), the crucial manipulation was performed between subjects, while in two others (Experiment 2, 1976; Experiment 1, 1977 ) it was performed within subjects. In the betweensubjects conditions, one group (story) was instructed to give a sentence using each word in a meaningful way and to make the sentences part of a continuing story. The subjects were not informed of the subsequent recall test. The other group (remember) was instructed to give a sentence for each word and to try to remember the words for a later recall test. The same manipulation was accomplished on a within-subjects basis by marking the even-numbered words with an $\mathrm{X}$ and the odd-numbered words with an 0 . Subjects were instructed to give a sentence for each word, using it in a meaningful way. In addition, they were instructed to try to remember the $\mathrm{X}$ words and to make the $\mathrm{O}$ words part of a continuing story.

In all four experiments more words were recalled in the story conditions than in the remember conditions. However, the differences were greater when the manipulation was within subjects than when it was between subjects. The first between-subjects experiment produced $32.40 \%$ and $24.74 \%$ recall for the story and remember conditions, respectively. Recall in Experiment 2 was $23.85 \%$ and $17.35 \%$ for the same conditions. The within-subjects experiments produced recalls of $48.35 \%$ (story) vs. $30.20 \%$ (remember) and $60.25 \%$ (story) vs. $38.05 \%$ (remember). The between-subjects conditions produced a difference of about $7 \%$, while the within-subjects conditions produced a difference of $20 \%$.

The Bellezza et al. between-groups experiments differed from ours in three ways, all of which may have resulted in less variability and therefore greater ability to detect small differences. First, they ran subjects individually; ours were run in small groups. Second, they presented the words individually on a memory drum; we used the whole-presentation method. Finally, they used a short (6- or 12-item) practice list prior to the main list; we did not use a practice list. It is possible that the advantage gained by using the narrative chaining technique without prior practice is quite small (about $7 \%$ ) and that the difference was hidden by the greater variability inherent in our procedures.

To explain the larger differences obtained between the story and remember groups in the within-subjects experiments, we return to the strategy most probably employed by subjects instructed to construct a continuing story. In order to make the story continuous, the subjects may have covertly rehearsed the preceding portions of their story while the remember words (those not in the story) were presented. Such a rehearsal strategy would reduce the effective study time available to the remember words and thus accentuate the difference in recall between the two types of words.

In conclusion, it appears that the advantage in recall afforded by use of the narrative chaining procedure may be found only when subjects have had practice with such a procedure or when use of such a strategy results in differential processing of list items. Whether an advantage results from deeper levels of processing, more organization, or both, is not clear. 


\section{REFERENCES}

Bellezza, F. S., Chessman, F. L. II, \& Reddy, B. G. Organization and semantic elaboration in free recall. Journal of Experimental Psychology: Human Learning and Memory, 1977, 3, 539-550.

Bellezza, F. S., Richards, D. L., \& Geiselman, R. E. Semantic processing and organization in free recall. Memory \& Cognition, 1976, 4, 415-421.

Bower, G. H., \& Clark, M. C. Narrative stories as mediators for serial learning. Psychonomic Science, 1969, 14, 181-182.

Craik, F. I. M., \& Lockhart, R. S. Levels of processing: A framework for memory research. Journal of Verbal Learning and Verbal Behavior, 1972, 11, 671-684.

Craik, F. I. M., \& Tulving, E. Depth of processing and the retention of words in episodic memory. Journal of Experimental Psychology: General, 1975, 104, 268-294.
Herrman, O. J., Geisler, F. V., \& Atrinson, R. C. The serial position function for lists learned by a narrative-story mnemonic. Bulletin of the Psychonomic Society, 1973, 2, 377-378.

Light. L. L., \& SchurR, S. C. Context effects in recognition memory: Item order and unitization. Journal of Experimental Psychology, 1973, 100, 135-140.

Paivio, A., Yuille, J. C., \& Madigan, S. Concreteness, imagery, and meaningfulness values for 925 nouns. Journal of Experimental Psychology Monograph Supplement, 1968, 76(1, pt. 2).

Santa, J. L., Ruskin, A. B., \& Yio, J. H. Mnemonic systems in free recall. Psychological Reports, 1973, 32, 1163-1170.

(Received for publication May 22, 1978.) 